\title{
BMJ Open Construction of the secondary care administrative records frailty (SCARF) index and validation on older women with operable invasive breast cancer in England and Wales: a cohort study
}

To cite: Jauhari Y, Gannon MR, Dodwell D, et al. Construction of the secondary care administrative records frailty (SCARF) index and validation on older women with operable invasive breast cancer in England and Wales: a cohort study. BMJ Open 2020;10:e035395. doi:10.1136/ bmjopen-2019-035395

- Prepublication history and additional material for this paper are available online. To view these files, please visit the journal online (http://dx.doi. org/10.1136/bmjopen-2019035395).

Received 04 November 2019 Revised 28 February 2020 Accepted 31 March 2020

Check for updates

(C) Author(s) (or their employer(s)) 2020. Re-use permitted under CC BY-NC. No commercial re-use. See rights and permissions. Published by BMJ.

For numbered affiliations see end of article.

Correspondence to

Yasmin Jauhari;

yjauhari@rcseng.ac.uk

\section{ABSTRACT}

Objectives Studies that use national datasets to evaluate the management of older women with breast cancer are often constrained by a lack of information on patient fitness. This study constructed a frailty index for use with secondary care administrative records and evaluated its ability to improve models of treatment patterns and overall survival in women with breast cancer.

Design Retrospective cohort study.

Participants Women aged $\geq 50$ years with oestrogen receptor (ER) positive early invasive breast cancer diagnosed between 2014 and 2017 in England.

Methods The secondary care administrative records frailty (SCARF) index was based on the cumulative deficit model of frailty, using International Statistical Classification of Diseases, Injuries and Causes of Death, 10th revision codes to define a set of deficits. The index was applied to administrative records that were linked to national cancer registry datasets. The ability of the SCARF index to improve the performance of regression models to explain observed variation in the rate of surgery and overall survival was evaluated using Harrell's c-statistic and decision curve analysis. External validation was performed on a dataset of similar women diagnosed in Wales.

Results The SCARF index captured 32 deficits that cover functional impairment, geriatric syndromes, problems with nutrition, cognition and mood, and medical comorbidities. In the English dataset $(n=67$ 925), the prevalence of frailty in women aged $50-69$, $70-79$ and $\geq 80$ years was $15 \%, 28 \%$ and $47 \%$, respectively. Adding a frailty measure to regression models containing age, tumour characteristics and comorbidity improved their ability to: (1) discriminate between whether a woman was likely to have surgery and (2) predict overall survival. Similar results were obtained when the models were applied to the Welsh cohort ( $n=4230)$.

Conclusion The SCARF index provides a simple and consistent method to identify frailty in population level data and could help describe differences in breast cancer treatments and outcomes.

\section{Strengths and limitations of this study}

- The secondary care administrative records frailty (SCARF) index was based on the cumulative deficit model of frailty and shares a similar construct to the Canadian Study of Health and Aging Frailty Index and the electronic Frailty Index.

- The study used population-based cohorts of women with breast cancer from England and Wales, with the English dataset being used to develop the SCARF index and the Welsh dataset being used for external validation.

- The deficits used in the SCARF index were defined using the International Statistical Classification of Diseases, Injuries and Causes of Death, 10th revision diagnosis codes recorded in the English and Welsh hospital administrative databases.

- A limitation of the study was its complete case analysis, with $16 \%$ of records excluded because of missing values in key variables.

- This study evaluated the SCARF index within a specific disease rather than the general population.

\section{INTRODUCTION}

UK-based population level studies have demonstrated wide variation in the treatment and outcomes of older women with early invasive breast cancer (EIBC). ${ }^{12}$ Differences in how health services manage older women with decreased levels of fitness are often cited as a key factor contributing to this variation. ${ }^{2}$ This has been difficult to investigate using routinely collected national datasets for breast cancer because they contain limited information on patient fitness. ${ }^{3}$ Studies have commonly adopted a measure of comorbidity burden (eg, Charlson Comorbidity Index $(\mathrm{CCI}))^{124}$ to account for different levels of fitness, but this is not ideal because ageing is a multidimensional concept that spans 
functional ability, physiology and social well-being. ${ }^{5}$ Therefore, in order to better understand the reasons for variation between older and younger women in treatments and outcomes for EIBC, measures that capture the multiple aspects of ageing are required.

The notion of biological age has evolved to describe the physiological differences among people of the same chronological age and is strongly linked to the concept of frailty. ${ }^{6}$ Frailty is described as a dynamic and heterogeneous manifestation of age-related decline in physiological reserve and increased vulnerability to stressors. ${ }^{8-10}$ While frailty is associated with comorbidity, it is recognised as a distinct geriatric syndrome that is potentially modifiable. ${ }^{5}$ However, there is no consensus on how to measure frailty and numerous instruments have been developed and used in different populations and healthcare settings. ${ }^{8} 1112$ The Comprehensive Geriatric Assessment (CGA) is one method that is widely recommended by geriatric professional bodies. ${ }^{6}{ }^{13}$ The CGA covers groups of domains: cognition/mood, nutrition, functional status, geriatric syndromes, comorbidity and polypharmacy. ${ }^{6}$ Each CGA domain is individually assessed for appropriate interventions, and because no single assessment method is defined for each domain, results from different settings may not be comparable. ${ }^{613}$ Various frailty instruments have also been developed for screening patients and identifying those who would benefit from a more comprehensive assessment, but a review of 28 highly cited instruments reported considerable heterogeneity between the measures and the thresholds used to categorise patients as frail or non-frail. ${ }^{14}$ For these reasons, it is unsurprising that population-based databases have been slow to incorporate the collection of frailty data items.

Various attempts have been made to create a frailty measure suitable for use within national datasets. Recently, in the UK, four frailty indices have been developed: two using primary care data (electronic Frailty Index (eFI) ${ }^{15}$ Q-frailty $)^{16}$ and two using administrative hospital data (frailty score ${ }^{17}$ and hospital frailty risk score) ${ }^{18}$ All four indices adopt the 'cumulative deficits' model of frailty, ${ }^{19}$ which conceptualises frailty as an increasing number of problems in different physiological systems that can present as clinical signs or symptoms, physical or cognitive impairments, or diseases. ${ }^{20}$ However, these indices are not easily applicable to the population-based datasets available from UK cancer registration services. For example, the calculation of the primary care Q-frailty index uses information on medication and biochemical results that are not collected in UK cancer registration datasets. ${ }^{16}$ Indices derived from administrative hospital datasets can be more readily applied to UK cancer registration data. Yet, current indices are limited in their coverage of frailty domains ${ }^{17}$ and may overestimate the prevalence of frailty due to a methodological assumption of a 'frail cluster' in hospital admissions. ${ }^{18}$ Additionally, the performance of these indices have not been evaluated in subgroups such as patients with cancer.
The aim of this study was to construct a frailty index using data available from the cancer registration services in England and Wales and evaluate its performance as a descriptor of biological age in women with breast cancer. In particular, the study evaluated the ability of the frailty index to improve the performance of regression models to explain: (1) patterns of primary surgery and (2) 3-year survival in women aged $\geq 50$ years with oestrogen receptor (ER) positive EIBC.

\section{METHODS}

\section{Data source}

The study was undertaken as part of the National Audit of Breast Cancer in Older Patients (NABCOP) ${ }^{21}$ and used pseudonymised patient-level datasets provided by the National Cancer Registration and Analysis Service for England and Wales Cancer Network for Wales. The linked datasets covered women aged $\geq 50$ years diagnosed by National Health Service (NHS) breast cancer units in England $(n=124)$ and Wales $(n=6)$ and provided data on patient demographics, tumour information, as well as treatment details. Additional information was provided by extracts from routine hospital admission databases for NHS hospitals (Hospital Episode Statistics (HES) for England and Patient Episode Database for Wales (PEDW) ). Full details of the NABCOP cohort are detailed in the 2019 annual report. ${ }^{3}$ Data quality and completeness of the variables used in analysis were comparable between English and Welsh datasets. ${ }^{3}$

\section{Study population and definitions}

This study used the NABCOP datasets to create a cohort of women aged $\geq 50$ years with new unilateral oestrogen receptor (ER-) positive early stage (Union for International Cancer Control (UICC) TNM staging classification, seventh edition: stage $1-3 a$ ) invasive breast cancer (International Statistical Classification of Diseases, Injuries and Causes of Death, 10th revision (ICD-10) ${ }^{22}$ code: C50) diagnosed between 1 January 2014 and 31 December 2017 in an NHS hospital in England or Wales $(\mathrm{n}=85210){ }^{3}$ Women with no linked hospital administrative dataset record $(\mathrm{n}=5543 ; 6.5 \%)$ or missing data for key analysis variables $(\mathrm{n}=7512 ; 8.6 \%)$ were excluded.

Information on patient demographics (age at diagnosis and deprivation) and tumour characteristics were obtained from the cancer registration datasets. Area-level socioeconomic deprivation was measured using the Index for multiple deprivation (IMD), with the IMD values for their geographical area in Wales ${ }^{23}$ and England ${ }^{24}$ converted to quintiles. Comorbidity burden was derived from the hospital administrative datasets using the Royal College of Surgeons Charlson Comorbidity Index (CCI), excluding malignancy, ${ }^{4}$ using a look back period of 2 years from date of breast cancer diagnosis. ${ }^{25}$

Primary surgery was defined as the first surgical procedure performed within 6 months of diagnosis, as recorded in HES or PEDW. Surgical procedures were described 
in the datasets using Office of Population Censuses and Surveys (OPCS) procedure codes, from which the study distinguished between breast conserving surgery (OPCS codes: B28, excluding B28.4 and B28.6) and mastectomy (any B27 code). Women were defined as not having surgical treatment if there was no record of these procedures in the 6 months after diagnosis in HES or PEDW.

Survival information was derived from the Civil Registration/Mortality data (previously known as the Office for National Statistics death register), which contains information on cause and date of death. Time to death was defined as the interval between date of diagnosis and date of death (in days). English women who were alive at the end of follow-up were censored on the final date of vital status assessment (27 January 2019). The final assessment date were not available for women in Wales, so women who were alive were censored on the day after the last reported date of death (19 September 2018). Median follow-up time was calculated in relation to the potential follow-up time of all women using reverse Kaplan-Meier estimate. $^{26}$

\section{Construction of the secondary care administrative records frailty (SCARF) index}

The cumulative deficit model of frailty ${ }^{19}$ was adopted as the basis for the index, with each frailty deficit defined using ICD-10 diagnosis codes. ${ }^{22}$ We chose to limit the derivation of the index to information available from hospital administrative datasets and not use information from cancer registration data, so that it can be applied to studies that only have access to routine hospital datasets. Hence, it was referred to as the SCARF index.

The initial stage of development involved a review of the deficits used in existing population-level frailty indices. ${ }^{15-1927} 28$ The review highlighted that the majority of indices adapted the original 92 deficits in the Canadian Study of Health and Aging Frailty Index. ${ }^{19} 27$ This approach was also used by the eFI,${ }^{15}$ which was developed and validated in the UK primary care setting ${ }^{1529} 30$ and which has been incorporated into the English general practitioner summary care record for clinical use. ${ }^{31}$ Consequently, the initial list for the SCARF index was based on the 36 deficits used by the eFI so that it shared a similar construction and thus may be a complementary instrument in the secondary care setting.

The subsequent stages of the development process are outlined below.

1. Identification of administrative data and ICD-10 codes for each frailty deficit.

Diagnostic codes for each deficit were identified through a search of the ICD-10 system and examination of other ICD-10 derived indices. ${ }^{17} 18$ This process was also informed by cross-referencing potential ICD10 codes with the primary care Read codes used in the eFI, although there is not a simple mapping between the two classifications. Two independent authors ( $\mathrm{YJ}$ and TR) inspected the final cohort of ICD-10 and administrative dataset codes for clinical validity.
Box 1 Inclusion criteria for deficits in the secondary care administrative dataset frailty (SCARF) index

Inclusion criteria for deficits in the SCARF index

1. Deficits describe a patient's health status rather than a reflection of the local service provisions in the secondary sector, for example, prolonged inpatient stay for rehabilitation.

2. The prevalence of a deficit increases with chronological age and does not saturate too early (the saturation point is defined as prevalence $>50 \%$ in persons aged $70+$ years).

3. The population prevalence of the deficit should be $>0.5 \%$ to avoid inclusion of rare or poorly recorded deficits.

4. As a group, deficits within the SCARF index should cover all the domains of the Comprehensive Geriatric Assessment (CGA).*

*Excluding polypharmacy.

2. Exploration of deficit prevalence within English patient cohort.

The initial SCARF index ICD-10 coding framework was applied to the HES records of the English women with breast cancer. The process involved checking the 20 diagnostic data fields in any patient record from the date of cancer diagnosis up to 2 years prior $^{18}{ }^{25}$ and enabled an initial estimate of the prevalence of each deficit to be derived. Each frailty deficit was then evaluated against a set of criteria for inclusion of the deficit in an index (Box 1), adapted from Searle $e t a l^{2}$ and Clegg $e t a l^{15}$ and those that failed to meet the criteria were dropped.

3. Calculation of the SCARF index and categories from the deficit profile.

The SCARF index for a patient was calculated as the number of deficits present divided by the total number in the index. The SCARF index was also stratified into four levels of fitness as follows: fit $=0-1$ deficit (index: $0-0.05$ ), mild frailty $=2-3$ deficits (index: 0.06-0.11), moderate frailty $=4-5$ deficits (index: $0.12-0.18$ ) and severe frailty $=6$ or more deficits (index: $\geq 0.19$ ).

\section{Evaluation of the SCARF index's performance}

Frailty is closely related to comorbidity but they are both independent concepts of ageing. ${ }^{5}$ In this study, the degree of association between the CCI (as a measure of comorbidity) and the SCARF index (as a measure of frailty) was evaluated using the Pearson's correlation coefficient. ${ }^{33}$ A correlation value of $r>0.8$ describes a strong positive correlation between two factors. ${ }^{34}$

The SCARF index was evaluated separately on women diagnosed in England and Wales due to the different constructs of administrative data sources. Within the English HES database, each record corresponds to the time a patient is under the care of the same consultant and contains up to 20 ICD-10 diagnosis codes (a single admission can consist of one or more consultant episodes). The Welsh PEDW database is not as rich in diagnostic information. Each record corresponds to a single admission and allows up to 14 ICD-10 codes to be stored. These differences between the two datasets was a 
Table 1 Baseline tumour characteristics of women diagnosed with ER-positive early invasive breast cancer between 2014 and 2017 in England and Wales by age group at diagnosis

\begin{tabular}{|c|c|c|c|c|c|c|c|c|}
\hline & \multicolumn{4}{|c|}{ England (internal validation) } & \multicolumn{4}{|c|}{ Wales (external validation) } \\
\hline & \multicolumn{3}{|c|}{ Age group at diagnosis (n (\%)) } & \multirow[b]{2}{*}{ Total } & \multicolumn{3}{|c|}{ Age group at diagnosis (n (\%)) } & \multirow[b]{2}{*}{ Total } \\
\hline & $50-69$ years & $70-79$ years & $\geq 80$ years & & $50-69$ years & $70-79$ years & $\geq 80$ years & \\
\hline \multicolumn{9}{|l|}{ Invasive grade } \\
\hline G1 & $9539(21)$ & $2490(17)$ & $1323(16)$ & 13352 & $770(27)$ & $208(21)$ & $69(16)$ & 1047 \\
\hline G2 & $26184(59)$ & $9304(63)$ & $5511(65)$ & 40999 & $1585(56)$ & $602(61)$ & $290(67)$ & 2477 \\
\hline \multicolumn{9}{|l|}{ Tumour size (T stage) } \\
\hline 1-20 mm (T0-1) & $29426(66)$ & $8314(56)$ & $3485(41)$ & 41225 & $1921(68)$ & $550(56)$ & $163(38)$ & 2634 \\
\hline 21-50 mm (T2) & 13505 (30) & $5947(40)$ & $4522(54)$ & 23974 & 779 (28) & 407 (41) & $242(56)$ & 1428 \\
\hline $51+\mathrm{mm}(\mathrm{T} 3)$ & $1693(4)$ & $601(4)$ & $432(5)$ & 2726 & $112(4)$ & $27(3)$ & $29(7)$ & 168 \\
\hline \multicolumn{9}{|l|}{ Nodal status ( $\mathrm{N}$ stage) } \\
\hline \multicolumn{9}{|l|}{ HER2 status } \\
\hline Positive & $4380(10)$ & $1215(8)$ & $676(8)$ & 6271 & $300(11)$ & $90(9)$ & $41(3)$ & 431 \\
\hline Negative & $37028(83)$ & $12543(84)$ & $6993(83)$ & 56564 & $2512(89)$ & $894(91)$ & $393(97)$ & 3799 \\
\hline Borderline & $3216(7)$ & $1104(7)$ & $770(9)$ & 5090 & - & & - & - \\
\hline \multicolumn{9}{|l|}{ IMD status } \\
\hline 1 - most deprived & $6812(15)$ & 2014 (14) & 1239 (15) & 10065 & $451(16)$ & $154(16)$ & $59(14)$ & 664 \\
\hline 2 & $8042(18)$ & $2548(17)$ & $1541(18)$ & 12131 & $524(19)$ & 175 (18) & $89(21)$ & 788 \\
\hline 3 & $9221(21)$ & 3107 (21) & $1809(21)$ & 14137 & $599(21)$ & $191(19)$ & 105 (24) & 895 \\
\hline 4 & $10412(23)$ & 3618 (24) & $1968(23)$ & 15998 & $573(20)$ & $211(21)$ & $77(18)$ & 861 \\
\hline Fit & $38682(87)$ & 10713 (72) & 4405 (52) & 53800 & 2507 (89) & 757 (77) & $232(53)$ & \\
\hline Mild frailty & 3897 (9) & $2224(15)$ & $1380(16)$ & 7501 & $216(8)$ & $131(13)$ & $89(21)$ & \\
\hline Moderate frailty & $1670(4)$ & 1367 (9) & $1461(17)$ & 4498 & $73(3)$ & $73(7)$ & $59(14)$ & \\
\hline Severe frailty & $375(1)$ & $558(4)$ & $1193(14)$ & 2126 & 16 (1) & $23(2)$ & $54(12)$ & \\
\hline
\end{tabular}

${ }^{*}$ Classification of the SCARF index: fit $=0-1$ deficit (index: $0-0.05$ ), mild frailty $=2-3$ deficits (index: $0.06-0.11$ ), moderate frailty $=4-5$ deficits (index: $0.12-0.18$ ) and severe frailty $=6$ or more deficits (index: $\geq 0.19$ ).

ER, Oestrogen receptor; HER2, human epidermal growth factor receptor 2; IMD, Index for multiple deprivation; SCARF, secondary care administrative records frailty.

key consideration in fitting the regression models to both the English and Welsh cohorts, because depth of coding available could affect the performance of a frailty index that relies on counting the cumulative number of deficits.

The explanatory power of the SCARF index was evaluated for two different outcome variables: the likelihood of surgery and survival. To examine the index's performance to predict the likelihood of women not receiving surgery for unilateral ER-positive EIBC, a series of multivariable multilevel logistic regression models were developed. The models included a random intercept for each NHS trust to account for the possible correlation between patient characteristics within organisations. Patient and tumour characteristics were included in the baseline model (model 1): deprivation quintile, tumour grade,human epidermal growth factor receptor 2 (HER2) status, tumour size (T stage) and nodal status (N stage). Models 2-6 included combinations of the CCI (comorbidity) and SCARF index (frailty) as measures of biological age and chronological age at diagnosis.

The association between the SCARF index and survival was evaluated using a series of Cox proportional hazards models that used the same six combinations of variables. Women who received surgery for ER-positive EIBC have 


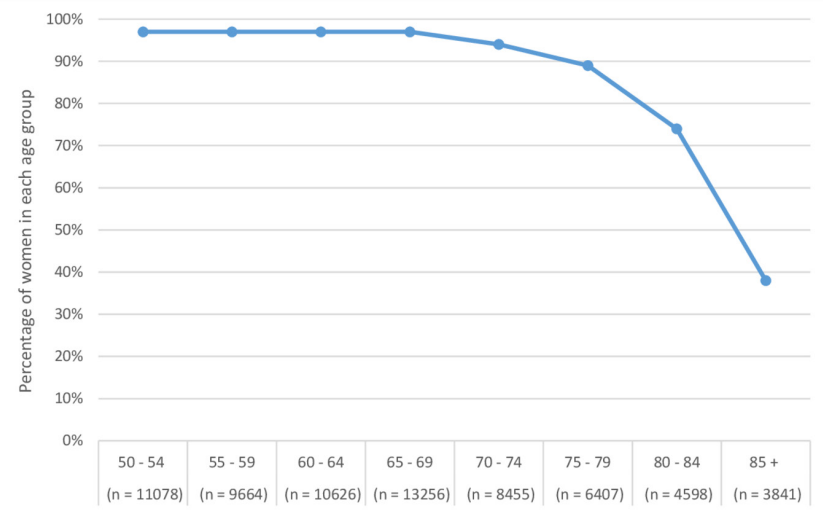

Age in years at diagnosis (total number of women)

Figure 1 Proportion of women receiving surgery for ERpositive early invasive breast cancer in England, between 2014 and 2017.

better survival than women who did not. ${ }^{35}$ Thus, the relationship between survival and surgery was explored by: (1) subgroups of women who did and did not have surgery and (2) using surgery as an explanatory variable in a Cox proportional hazards model for all women. The study specifically focused on 3-year survival because recommendations by the International Society of Geriatric Oncology and European Society of Breast Cancer Specialists for older women with breast cancer state that 'primary endocrine therapy (PET) should only be offered to elderly individuals with ER-positive tumours who have a short estimated life-expectancy (<2-3 years), who are considered unfit for surgery after optimization of medical conditions..., 36

The performance of the models was evaluated in terms of their discrimination using Harrell's c-statistic, ${ }^{37}$ which corresponds to the area under the curve. A c-statistic value of 0.5 indicates that the model's discriminative power is similar to chance, and 1.0 suggests perfect model discrimination. Their relative performance was also assessed using decision curve analysis (DCA). This method is based on evaluating the 'net-benefit' of model predictions across of range of possible decision thresholds that reflect how a patient might weigh the risk of harm associated with a false positive result (compared with a true positive result).$^{38}{ }^{39}$ Models with a better performance have a greater net benefit across all thresholds of probability.

A similar methodology was applied to externally validate the SCARF index using data on women diagnosed in Wales. Analyses were conducted using Stata V.15.1. All statistical tests were two sided.

\section{RESULTS}

\section{Frailty index development and internal validation}

The study cohort for England contained 67925 women aged $\geq 50$ years diagnosed with unilateral ER-positive EIBC between 1 January 2014 and 31 December 2017. Their clinical and pathological characteristics at diagnosis are summarised by age group in table 1 . The majority of women had grade 2, HER2-negative EIBC with no regional lymph node involvement (N0) and the proportion of women who presented with larger tumours (21-50 $\mathrm{mm}, \mathrm{T} 2)$ increased with age.

The development process resulted in an index containing 32 deficits defined using used 356 ICD-10 codes (online supplementary table 1 ). The initial review identified potential diagnostic codes for all 36 deficits, with the exception of polypharmacy. This deficit was therefore dropped because medication prescription records are unavailable in HES and PEDW. The evaluation of the remaining 35 deficits against the criteria in Box 1 led to several complementary deficits being grouped together ('housebound' and 'requirement for care') and one deficit ('sleep disturbance') being dropped due to a low prevalence. The mean index and 99th centile values for the SCARF index in the English cohort were 0.05 and 0.30 , respectively, and had positive correlation with the CCI $(r=0.76)$. The SCARF index also revealed an increasing burden of frailty across the CGA domains (problems with cognition/mood, nutrition or ability to function daily, geriatric syndromes and comorbidity), ${ }^{6}$ with older age (online supplementary table 2 ).

\section{Association of the SCARF index with likelihood of surgery}

Among the 67975 women with ER-positive EIBC, 61813 $(91 \%)$ underwent surgery. The rate of surgery decreased with older age (figure 1), but age was only one of the patient and tumour factors associated with the likelihood of not receiving surgery (table 2). When considered in separate models, greater burden of comorbidity (model 2) or frailty (model 3) increased the likelihood of women not receiving surgery. Both these factors remained predictors after baseline tumour factors and chronological age were accounted for. In particular, frailty was more strongly associated with reduced likelihood of receiving surgery compared with comorbidity (models 4 and 6 ). The model that included comorbidity and frailty as biological age measures together with chronological age had the greatest discrimination, with a c-statistic of 0.858 (table 2, model 6). A similar pattern of improving performance is shown in the results of the decision curve analysis (figure 2). All models had a greater net benefit than the two default decision strategies of no women or all women having surgery, but there was considerable improvement when the biological age factors (frailty and comorbidity) were included to the models (with or without chronological age).

\section{Association of the SCARF index with survival}

The survival analysis had a median follow-up of 35 months (IQR: 24 and 46 months). Irrespective of age, the rate of early death was higher among women who did not have surgery compared with those who did (figure 3). In both circumstances, older chronological age and high-risk tumour characteristics, such as higher grade and presence of nodal metastasis, were predictive of worse 3-year overall survival (model 5). The inclusion of biological age 


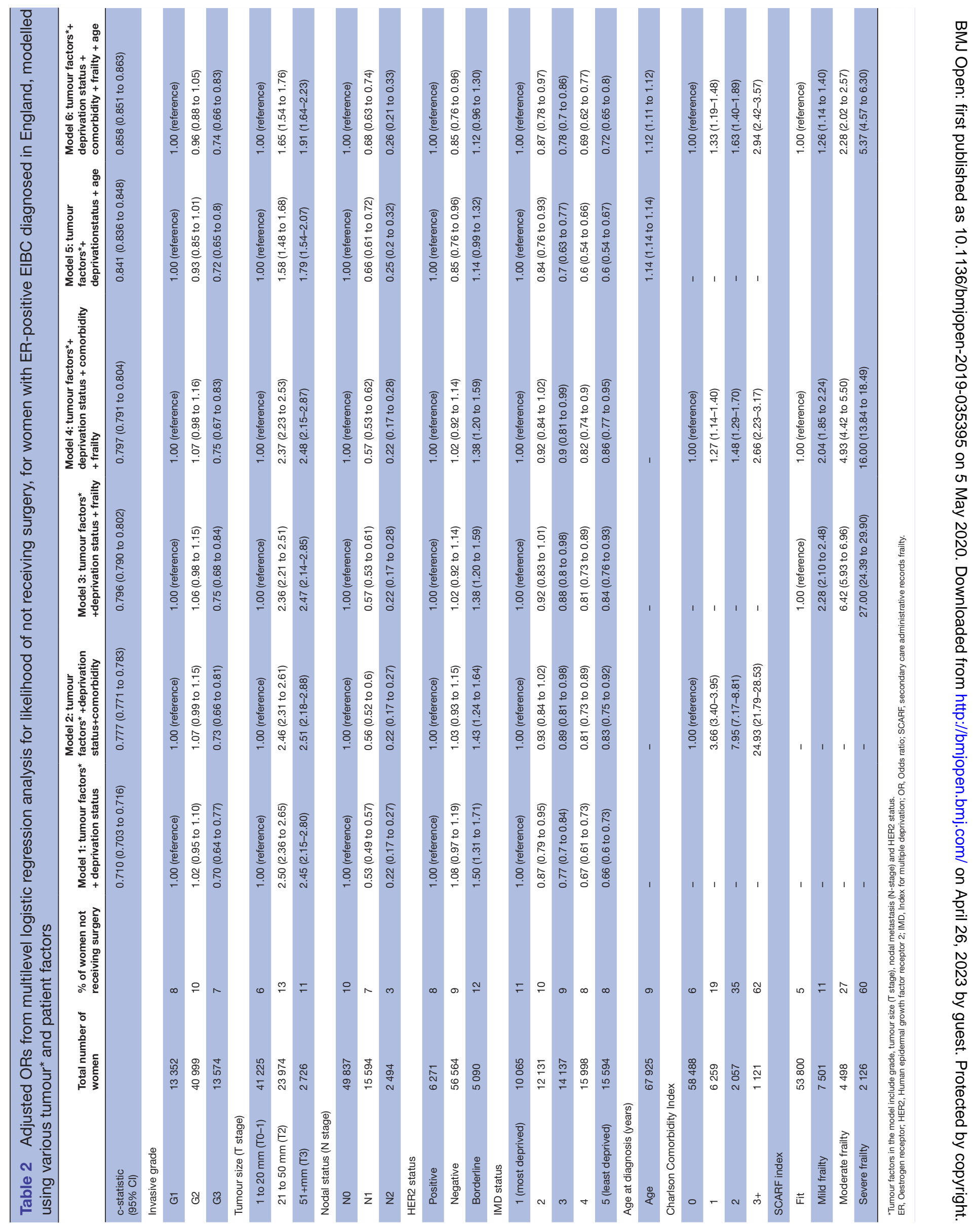



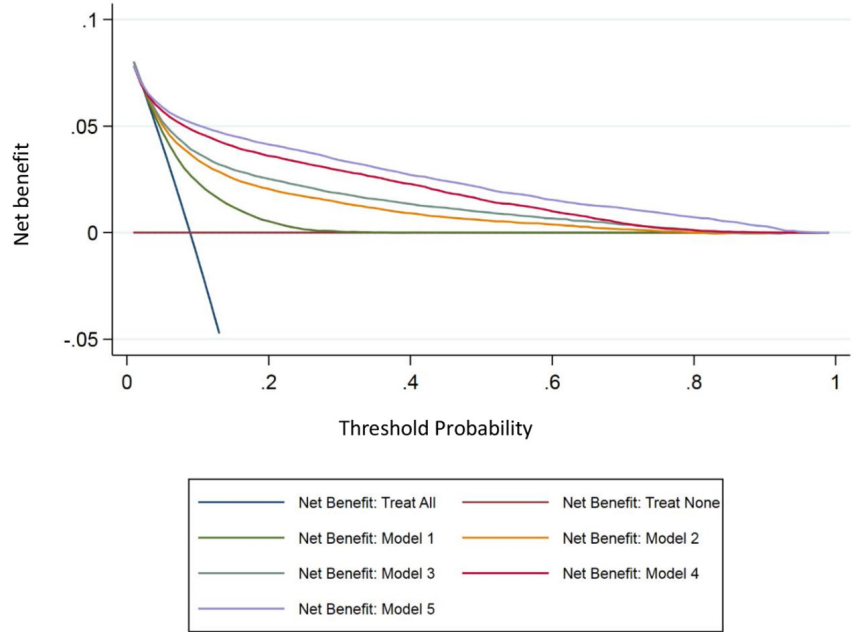

Figure 2 Decision curve for likelihood of not receiving surgery for ER-positive EIBC in England: a comparison of models with tumour factors and deprivation status only (model 1 ) and models with the addition of Charlson Comorbidity Index (CCl) (model 2), secondary care administrative records frailty (SCARF) index (model 3), $\mathrm{CCl}$ and SCARF index (model 4), chronological age only (model 5) and $\mathrm{CCl}$, SCARF index and age (model 6).

measures (model 6) improved the model performance, increasing the c-statistic from 0.750 to 0.770 in women who had surgery and from 0.638 to 0.663 in women who did not have surgery and from 0.843 to 0.852 in all women (table 3). Similar to previous, this full model (model 6) demonstrated the best discrimination. In the DCA, model 6 with both measures of biological and chronological age again produced the best overall performance (figure 4).

Table 4 describes the relationships between overall survival and the tumour and patient factors for women with ER-positive EIBC as estimated in model 6. As expected, worse survival was associated with high-risk tumour characteristics (eg, higher grade and presence of nodal metastasis), a burden of comorbidity or frailty, advanced age and not having surgery.

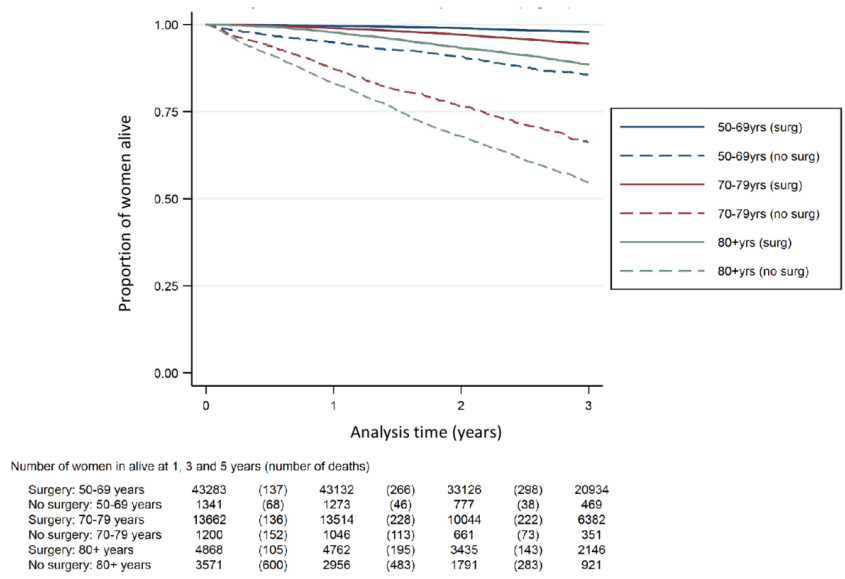

Figure 3 Observed survival for women with ER-positive EIBC diagnosed between 2014 and 2017 in England by age group at diagnosis and receipt of surgery. EIBC, early invasive breast cancer.

\section{External validation of the SCARF index}

The cohort of Welsh patients consisted of 4230 women aged $\geq 50$ years, diagnosed with unilateral ER-positive EIBC between January 2014 and December 2017. The median age was 66 years (range 50-100): 64\% ( $\mathrm{n}=2$ 812) were aged 50-69 years, 22\% ( $\mathrm{n}=984)$ were aged $70-79$ years and $14 \% \quad(n=434)$ were aged $\geq 80$ years. Baseline patient and tumour characteristics were similar between the English and Welsh cohorts (table 1). Specifically, although the calculations of the CCI and SCARF index for Welsh patients could only use records with 14 diagnostic fields (compared with the 20 diagnostic fields in HES), there was a similar prevalence of comorbidity and frailty in the two cohorts. The mean SCARF index and 99 th centile values were 0.03 and 0.31 , respectively.

There were 3913 (93\%) Welsh women who received surgical treatment for EIBC. The associations between the tumour and patient factors and the likelihood of a woman not having surgery were similar to those observed in English cohort (online supplementary table 3). In particular, the inclusion of biological age factors (comorbidity and frailty) and chronological age in a model improved the ability of the model to discriminate between women who did and did not receive surgery (corresponding increase in c-statistic from 0.830 in model 5 , to 0.848 in model 6 ). A similar effect was observed for the overall survival of the women diagnosed in Wales (corresponding increase in c-statistic from 0.837 in model 5 , to 0.841 for model 6) (table 3).

\section{DISCUSSION}

Frailty is a commonly used descriptor of biological age in research studies, but measurements of frailty have yet to be incorporated in routinely collected healthcare data. ${ }^{12}$ This study describes the construction of the SCARF index based on the widely adopted cumulative deficits model of frailty, as used in the $\mathrm{eFI}^{15}$ and CHSA frailty index. ${ }^{40}$ The SCARF index was designed for use in analyses of population-level cancer registration data and can also be used in hospital administrative datasets. In this population of women with breast cancer, the index demonstrated good face validity by producing the expected increasing prevalence of frailty among older patients, both overall and within the CGA domains. It also demonstrated a strong association with both patterns of surgical practice and patient survival when added to models that also contained age and comorbidity.

Standardising methods to measure frailty is particularly important to reliably estimate the prevalence of frailty and to understand its influence on treatment patterns and outcomes. This study focused on women with breast cancer because it is the most common type of female cancer, and older chronological age is a well-established risk-factor for disease development. ${ }^{41}$ The majority of women present with early stage breast cancer ${ }^{3}$ and while this may represent a low-risk group with respect to their breast cancer, the cohort would contain the typical 
Table 3 Harrell's c-statistic for discrimination ability of Cox proportional hazards models on 3-year overall survival of women aged $50+$ years with ER-positive EIBC by country of diagnosis

\begin{tabular}{|c|c|c|c|c|c|c|c|}
\hline \multirow[b]{3}{*}{$\begin{array}{l}\text { Model } \\
\text { no. }\end{array}$} & \multirow[b]{3}{*}{$\begin{array}{l}\text { Factors included in the cox regression model for } 3 \text { year } \\
\text { survival }\end{array}$} & \multicolumn{6}{|c|}{ Harrell's c-statistic for cox proportional hazards survival model } \\
\hline & & \multicolumn{3}{|c|}{ England (internal validation) } & \multicolumn{3}{|c|}{ Wales (external validation) } \\
\hline & & $\begin{array}{l}\text { In all } \\
\text { women* }\end{array}$ & $\begin{array}{l}\text { In women who } \\
\text { did receive } \\
\text { surgery }\end{array}$ & $\begin{array}{l}\text { In women who } \\
\text { did not receive } \\
\text { surgery }\end{array}$ & $\begin{array}{l}\text { In all } \\
\text { women* }\end{array}$ & $\begin{array}{l}\text { In women who } \\
\text { did receive } \\
\text { surgery }\end{array}$ & $\begin{array}{l}\text { In women who } \\
\text { did not receive } \\
\text { surgery }\end{array}$ \\
\hline 1 & $\begin{array}{l}\text { Invasive grade, T stage, N stage, HER2 status, IMD status } \\
\text { (+receipt of surgery*) }\end{array}$ & 0.804 & 0.669 & 0.552 & 0.782 & 0.634 & 0.653 \\
\hline 2 & $\begin{array}{l}\text { Invasive grade, T stage, N stage, HER2 status, IMD status+CCI } \\
\text { (+receipt of surgery*) }\end{array}$ & 0.824 & 0.708 & 0.618 & 0.802 & 0.664 & 0.679 \\
\hline 3 & $\begin{array}{l}\text { Invasive grade, T stage, N stage, HER2 status, IMD } \\
\text { status+SCARF index (+receipt of surgery*) }\end{array}$ & 0.828 & 0.718 & 0.625 & 0.804 & 0.667 & 0.691 \\
\hline 4 & $\begin{array}{l}\text { Invasive grade, T stage, N stage, HER2 status, IMD } \\
\left.\text { status+CCI+SCARF index (+receipt of surgery }{ }^{\star}\right)\end{array}$ & 0.829 & 0.719 & 0.628 & 0.805 & 0.673 & 0.690 \\
\hline 5 & $\begin{array}{l}\text { Invasive grade, T stage, N stage, HER2 status, IMD status+age } \\
\text { (+receipt of surgery*) }\end{array}$ & 0.842 & 0.750 & 0.638 & 0.837 & 0.723 & 0.703 \\
\hline 6 & $\begin{array}{l}\text { Invasive grade, T stage, N stage, HER2 status, IMD } \\
\text { status+CCI+SCARF index+age (+receipt of surgery*) }\end{array}$ & 0.852 & 0.770 & 0.663 & 0.841 & 0.734 & 0.715 \\
\hline
\end{tabular}

*The receipt of surgery was only factored in the model that included all women.

$\mathrm{CCI}$, Charlson Comorbidity Index; EIBC, early invasive breast cancer; HER2, Human epidermal growth factor receptor 2; IMD, Index for multiple deprivation; SCARF, secondary care administrative records frailty.

spectrum of frailty seen among older women. In addition, breast cancer trials that focus on older women are often unsuccessful due to poor accrual, ${ }^{42}$ thus routinely collected data are an increasingly valuable research resource in this population.

Surgical resection is the standard of care for women with EIBC. ${ }^{43}$ Specifically, in women with ER-positive EIBC, surgery offers survival outcomes that are superior to primary endocrine therapy. ${ }^{35}$ However, variation in the rate of primary surgery and survival, between older and younger women with ER-positive EIBC is widely reported. $^{123544}$ The ability to distinguish between comorbidity and frailty is clinically relevant because these factors can be optimised, and potentially reversed (in the case of frailty), to improve rates of surgery and survival in women with EIBC. Another benefit of assessing frailty might also be the identification of older women who are sufficiently fit to be offered more aggressive breast cancer treatments. In time, we expect that an appropriate frailty instrument will be adopted within clinical practice, the results of which could be entered into cancer registration datasets. ${ }^{45}$ However, until then, population-based analyses that use national cancer datasets will be limited to using tools like the SCARF index to improve understanding of the influence of frailty on treatment patterns and survival. This is important for improving the quality of breast cancer care and for the education of health professionals. Clinicians can be inconsistent in estimating the life expectancy of older women with a higher burden of comorbidity and frailty, which subsequently influences their willingness to advocate surgery for these women. ${ }^{46}$

There are several strengths to this study. First, the SCARF index was constructed using solely ICD-10 diagnosis codes and can therefore be widely applied to other population level datasets. Moreover, the index produced similar results from using data from HES and PEDW although they differed in the number of available diagnostic fields
Women who received surgery
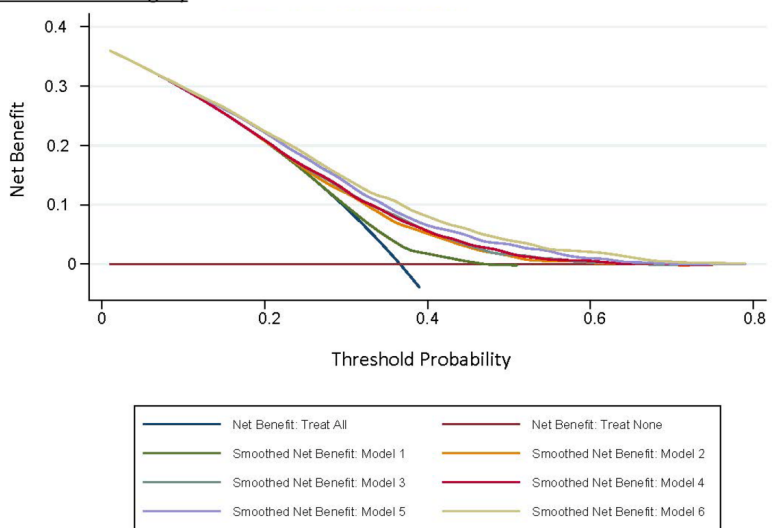

Women who did not receive surgery
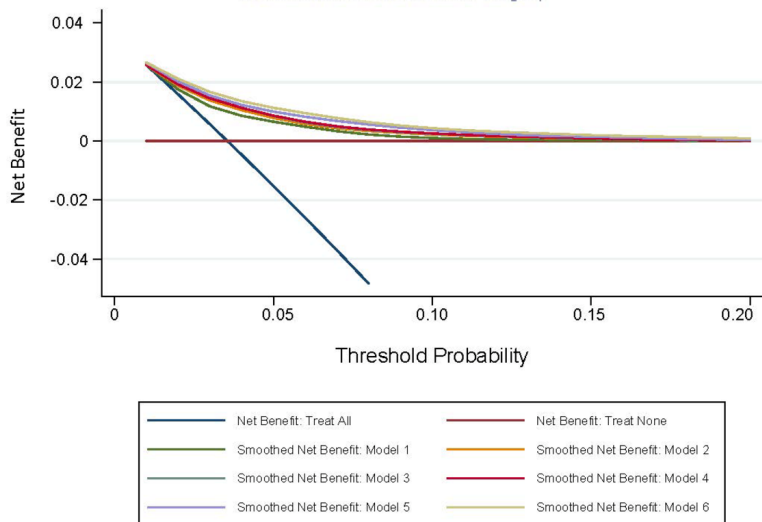

Figure 4 Decision curve for likelihood of survival in women who did and who did not receive surgery for ER-positive EIBC in England: comparison of models with tumour characteristics only (model 1) and models with the addition of Charlson Comorbidity Index (CCI) (model 2), secondary care administrative records frailty (SCARF) index (model 3), $\mathrm{CCl}$ and SCARF index (model 4), chronological age only (model 5) and $\mathrm{CCl}$, SCARF index and age (model 6). EIBC, early invasive breast cancer. 
Table 4 Cox proportional hazards survival analysis (model 6) for 3-year overall survival in women aged $\geq 50$ years with ERpositive early invasive breast cancer in England between 2014 and 2017

\begin{tabular}{|c|c|c|c|c|c|}
\hline & $\begin{array}{l}\text { Total number of } \\
\text { women }\end{array}$ & $\begin{array}{l}\% \text { women alive at } \\
3 \text { years }\end{array}$ & Adjusted OR & $95 \% \mathrm{Cl}$ & $P$ value \\
\hline Age at diagnosis (years) & 67925 & 94 & 1.06 & 1.05 to 1.06 & $<0.001$ \\
\hline \multicolumn{6}{|l|}{ Invasive grade } \\
\hline G1 & 13352 & 96 & 1 & & $<0.001$ \\
\hline G2 & 40999 & 94 & 1.05 & 0.96 to 1.16 & \\
\hline G3 & 13574 & 91 & 1.64 & 1.47 to 1.83 & \\
\hline \multicolumn{6}{|l|}{ Tumour size (T stage) } \\
\hline 1-20 mm (T0-1) & 41225 & 95 & 1 & & $<0.001$ \\
\hline 21-50 mm (T2) & 23974 & 90 & 1.31 & 1.22 to 1.41 & \\
\hline $51+\mathrm{mm}(\mathrm{T} 3)$ & 2726 & 89 & 1.69 & 1.48 to 1.93 & \\
\hline \multicolumn{6}{|c|}{ Nodal metastasis (N-stage) } \\
\hline NO & 49837 & 94 & 1 & & $<0.001$ \\
\hline $\mathrm{N} 1$ & 15594 & 93 & 1.39 & 1.29 to 1.50 & \\
\hline N2 & 2494 & 89 & 2.16 & 1.88 to 2.49 & \\
\hline \multicolumn{6}{|l|}{ HER2 status } \\
\hline Positive & 6271 & 92 & 1 & & $<0.001$ \\
\hline Negative & 56564 & 94 & 0.81 & 0.73 to 0.90 & \\
\hline Borderline & 5090 & 92 & 0.82 & 0.71 to 0.95 & \\
\hline \multicolumn{6}{|l|}{ Deprivation status } \\
\hline 1 (most deprived) & 10065 & 91 & 1 & & $<0.001$ \\
\hline 2 & 12131 & 93 & 0.86 & 0.78 to 0.95 & \\
\hline 3 & 14137 & 94 & 0.74 & 0.67 to 0.82 & \\
\hline 4 & 15998 & 94 & 0.77 & 0.70 to 0.85 & \\
\hline 5 (least deprived) & 15594 & 95 & 0.67 & 0.61 to 0.75 & \\
\hline \multicolumn{6}{|c|}{ Charlson Comorbidity Index } \\
\hline 0 & 58488 & 96 & 1 & & $<0.001$ \\
\hline 1 & 6259 & 87 & 1.25 & 1.11 to 1.40 & \\
\hline 2 & 2057 & 77 & 1.41 & 1.22 to 1.62 & \\
\hline $3+$ & 1121 & 59 & 1.86 & 1.59 to 2.18 & \\
\hline \multicolumn{6}{|c|}{ Secondary care administrative records frailty index index } \\
\hline Fit & 53800 & 96 & 1 & & $<0.001$ \\
\hline Mild frailty & 7501 & 92 & 1.22 & 1.09 to 1.36 & \\
\hline Moderate frailty & 4498 & 82 & 1.44 & 1.27 to 1.64 & \\
\hline Severe frailty & 2126 & 61 & 1.63 & 1.41 to 1.89 & \\
\hline \multicolumn{6}{|l|}{ Surgery } \\
\hline No & 6112 & 64 & 1 & & $<0.001$ \\
\hline Yes & 61813 & 96 & 0.21 & 0.20 to 0.23 & \\
\hline
\end{tabular}

${ }^{*} \mathrm{P}$ value from $\mathrm{z}$-test for continuous variables and Wald test for categorical variables.

(20 and 14, respectively). Second, the deficits within the SCARF index were based on those contained in two widely used frailty indices (eFI and CHSA) that have been validated using community populations. Last, the study demonstrated how the influences of chronological and biological age measures improve the explanatory power of models for two important patient outcomes.
There are also some study limitations. First, calculating the SCARF index relies on the accurate coding of patient diagnoses within administrative datasets. Conditions such as pressure ulcers and urinary incontinence were previously poorly recorded in administrative datasets, but there has been improvement in data quality over time and during the study period. ${ }^{47}$ The SCARF index also 
demonstrated an increasing prevalence of frailty with age, both as an overall score and within the CGA domains, which are similar to previous reports. Second, the calculations of the SCARF index deficits used data from 2 years prior to the date of diagnosis. This method is similar to published studies ${ }^{18}$ and unavoidable due to the static nature of administrative data. Given the potentially reversible nature of frailty, this might introduce some misclassification error. Third, the SCARF index was constructed using data from a female cohort with ER-positive EIBC. The study was restricted to describing frailty within a specific disease rather than the general population. Nonetheless, this study uses a method of index construction that has proven effective in many other population groups, and there is no reason to suspect the SCARF index measures a condition-specific form of frailty. We note that the widely used CCI was initially developed in a cohort of women with primary breast cancer. ${ }^{48}$ Finally, the results were based on a complete case analysis, with $6.5 \%$ of records dropped because they could not be linked to a HES/PEDW record and another $8.8 \%$ of records dropped because of missing values (mostly related to missing HER2 status (8.6\%)). This might introduce bias in the observed relationship between the SCARF index and the outcomes, but the effect is likely to be small. The distributions of age at diagnosis were similar among included and excluded patients, suggesting the analysis cohort retained a full spectrum of frailty.

It is important that further work is undertaken to extend the evaluation of the SCARF index. This includes the application of this index on different cohorts of patients with breast cancer or another cancer to examine the influence of frailty on disease presentation and uptake of adjuvant therapies. Future work also includes assessing the agreement between the SCARF index and other frailty assessment tools. One option is to compare it against the Clinical Frailty Scale, ${ }^{3}$ which will become part of routinely collected breast cancer data in England from $2020{ }^{45}$ Opportunities to triangulate between measures of frailty using hospital level (SCARF index) and primary care level data (eFI) should also be explored.

\section{Author affiliations}

${ }^{1}$ Clinical Effectiveness Unit, Royal College of Surgeons of England, London, UK

${ }^{2}$ Department of Health Services Research and Policy, London School of Hygiene \&

Tropical Medicine, London, UK

${ }^{3}$ Nuffield Department of Population Health, Oxford University, Oxford, UK

${ }^{4}$ Department of Breast Surgery, St James's University Hospital, Leeds, UK

${ }^{5}$ National Cancer Registration and Analysis Service, Public Health England, London, UK

${ }^{6}$ Centre for Surgical Research, Bristol Medical School: Population Health Sciences, University of Bristol, Bristol, UK

${ }^{7}$ Department of Cardiovascular Sciences and NIHR Leicester Biomedical Research Centre, University of Leicester, Leicester, UK

${ }^{8}$ St George's University of London, London, UK

\section{Twitter Yasmin Jauhari @aj_yasmin}

Contributors YJ: study concept and design, data acquisition management, statistical analysis and results interpretation and involvement in all stages of the manuscript development; MRG: study design, data acquisition and management, statistical analysis and results interpretation and involvement in all stages of manuscript development; DD and KH: study concept and design, data acquisition, interpretation of the results and involvement in all stages of manuscript development; KC: data acquisition, interpretation of the results and involvement in manuscript editing and review; JM: data acquisition and management, interpretation of the results and involvement in manuscript editing and review; CT: interpretation of the results and involvement in all stages of manuscript development; TR: study design, interpretation of the results and involvement in all stages of manuscript development; SS-KT and RP: interpretation of the results and involvement in manuscript editing and review; DAC: study concept and design, data acquisition and management, statistical analysis and results interpretation and involvement in all stages of the manuscript development. All authors have approved the final version of this article and agree to be accountable for all aspects of the work.

Funding This study was undertaken as part of the work by the National Audit of Breast Cancer in Older Patients. The Audit is commissioned by the Healthcare Quality Improvement Partnership (HQIP) as part of the National Clinical Audit and Patient Outcomes Programme and funded by NHS England and the Welsh Government (www.hqip.org.uk/national-programmes). The authors had full independence from the Healthcare Quality Improvement Partnership. The aim of National Audit of Breast Cancer in Older Patients (NABCOP) is to evaluate the care of older women with breast cancer in England and Wales, and support NHS providers to improve the quality of hospital care for these women. More information can be found at: www.nabcop.org.uk

Disclaimer Neither HQIP nor the funders had any involvement in the study design; in the collection, analysis and interpretation of data; in the writing of the report; or in the decision to submit the article for publication.

Competing interests None declared.

Patient and public involvement Patients and/or the public were not involved in the design, or conduct, or reporting, or dissemination plans of this research.

Patient consent for publication Not required.

Ethics approval The study is exempt from UK National Research Ethics Committee approval as it involved secondary analysis of an existing dataset of anonymised data. The NABCOP has approval for processing health care information under Section 251 (reference number: 16/CAG/0079) for all National Health Service (NHS) patients aged 50 years and over diagnosed with breast cancer in England and Wales. Data for this study are based on patient-level information collected by the NHS, as part of the care and support of patients with cancer. The data is collated, maintained and quality assured by the National Cancer Registration and Analysis Service, which is part of Public Health England.

Provenance and peer review Not commissioned; externally peer reviewed.

Data availability statement No data are available. № additional data available.

Open access This is an open access article distributed in accordance with the Creative Commons Attribution Non Commercial (CC BY-NC 4.0) license, which permits others to distribute, remix, adapt, build upon this work non-commercially, and license their derivative works on different terms, provided the original work is properly cited, appropriate credit is given, any changes made indicated, and the use is non-commercial. See: http://creativecommons.org/licenses/by-nc/4.0/.

\section{ORCID iD}

Yasmin Jauhari http://orcid.org/0000-0002-4761-8655

\section{REFERENCES}

1 Bates T, Evans T, Lagord C, et al. A population based study of variations in operation rates for breast cancer, of comorbidity and prognosis at diagnosis: failure to operate for early breast cancer in older women. Eur J Surg Oncol 2014;40:1230-6.

2 Richards P, Ward S, Morgan J, et al. The use of surgery in the treatment of ER+ early stage breast cancer in England: variation by time, age and patient characteristics. Eur J Surg Oncol 2016;42:489-96.

3 Healthcare Quality Improvement Partnership (HQIP). National audit of breast cancer in older patients (NABCOP): 2019 annual report 2019.

4 Armitage JN, van der Meulen JH. Royal College of surgeons comorbidity consensus $\mathrm{G}$. identifying co-morbidity in surgical patients using administrative data with the Royal College of surgeons Charlson score. Br J Surg 2010;97:772-81. 
5 Fried LP, Ferrucci L, Darer J, et al. Untangling the concepts of disability, frailty, and comorbidity: implications for improved targeting and care. J Gerontol A Biol Sci Med Sci 2004;59:M255-63.

6 Wildiers $\mathrm{H}$, Heeren P, Puts M, et al. International Society of geriatric oncology consensus on geriatric assessment in older patients with cancer. J Clin Oncol 2014;32:2595-603.

7 PACE participants, Audisio RA, Pope D, et al. Shall we operate? preoperative assessment in elderly cancer patients (PACE) can help. A SIOG surgical task force prospective study. Crit Rev Oncol Hematol 2008:65:156-63.

8 Xue Q-L. The frailty syndrome: definition and natural history. Clin Geriatr Med 2011;27:1-15.

9 Campbell AJ, Buchner DM. Unstable disability and the fluctuations of frailty. Age Ageing 1997;26:315-8.

10 Gill TM, Gahbauer EA, Allore HG, et al. Transitions between frailty states among community-living older persons. Arch Intern Med 2006;166:418.

11 Clegg A, Young J, lliffe S, et al. Frailty in elderly people. The Lancet 2013;381:752-62.

12 de Vries NM, Staal JB, van Ravensberg CD, et al. Outcome instruments to measure frailty: a systematic review. Ageing Res Rev 2011;10:104-14.

13 British Geriatric Society. Comprehensive assessment of the frail older patient, 2019. Available: https://www.bgs.org.uk/resources/resourceseries/comprehensive-geriatric-assessment-toolkit-for-primary-carepractitioners

14 Buta BJ, Walston JD, Godino JG, et al. Frailty assessment instruments: systematic characterization of the uses and contexts of highly-cited instruments. Ageing Res Rev 2016;26:53-61.

15 Clegg A, Bates C, Young J, et al. Development and validation of an electronic frailty index using routine primary care electronic health record data. Age Ageing 2016;45:353-60.

16 Hippisley-Cox J, Coupland C. Development and validation of QMortality risk prediction algorithm to estimate short term risk of death and assess frailty: cohort study. BMJ 2017;358:j4208.

17 Soong J, Poots AJ, Scott S, et al. Developing and validating a risk prediction model for acute care based on frailty syndromes. BMJ Open 2015;5:e008457.

18 Gilbert T, Neuburger J, Kraindler J, et al. Development and validation of a hospital frailty risk score focusing on older people in acute care settings using electronic Hospital records: an observational study. Lancet 2018;391:1775-82.

19 Mitnitski AB, Mogilner AJ, Rockwood K. Accumulation of deficits as a proxy measure of aging. ScientificWorldJournal 2001;1:323-36.

20 Rockwood K, Mitnitski A. Frailty in relation to the accumulation of deficits. J Gerontol A Biol Sci Med Sci 2007;62:722-7.

21 The National audit of breast cancer in older patients. Available: https://www.nabcop.org.uk/

22 World Health Organisation. International classification of diseases and health related problems. 10th edn, 1992.

23 Llywodraeth Cymru Welsh Government. Welsh index of multiple deprivation, 2014. Available: https://gov.wales/statistics-andresearch/welsh-index-multiple-deprivation/?lang=en

24 Communities and Local Government. The English indices of deprivation, 2015. Available: https://www.gov.uk/government/ statistics/english-indices-of-deprivation-2015

25 Preen DB, Holman C D'Arcy J, Spilsbury K, et al. Length of comorbidity lookback period affected regression model performance of administrative health data. J Clin Epidemiol 2006;59:940-6.

26 Clark TG, Bradburn MJ, Love SB, et al. Survival analysis Part I: basic concepts and first analyses. Br J Cancer 2003;89:232-8.

27 Mitnitski AB, Mogilner AJ, MacKnight C, et al. The accumulation of deficits with age and possible invariants of aging. The scientific world 2002;2.
28 Soong JTY, Poots AJ, Bell D. Finding consensus on frailty assessment in acute care through Delphi method. BMJ Open 2016;6:e012904.

29 Brundle C, Heaven A, Brown L, et al. Convergent validity of the electronic frailty index. Age and Ageing 2018:afy162-afy.

30 Bottle A, Kim D, Hayhoe B, et al. Frailty and co-morbidity predict first hospitalisation after heart failure diagnosis in primary care: population-based observational study in England. Age Ageing 2019;48:347-54.

31 NHS England. Updated guidance on supporting routine frailty identification and frailty care through the GP contract 2017/8, 2017. Available: https://www.england.nhs.uk/publication/supportingroutine-frailty-identification-and-frailty-through-the-gp-contract20172018/

32 Searle SD, Mitnitski A, Gahbauer EA, et al. A standard procedure for creating a frailty index. BMC Geriatr 2008;8:24.

33 Mukaka MM. Statistics corner: a guide to appropriate use of correlation coefficient in medical research. Malawi Med $\mathrm{J}$ 2012;24:69-71.

34 Evans JD. Straightforward statistics for the behavioral sciences. Pacific Grove, CA: Brooks/Cole Publishing, 1996.

35 Ward SE, Richards PD, Morgan JL, et al. Omission of surgery in older women with early breast cancer has an adverse impact on breast cancer-specific survival. Br J Surg 2018;105:1454-63.

36 Biganzoli L, Wildiers H, Oakman C, et al. Management of elderly patients with breast cancer: updated recommendations of the International Society of geriatric oncology (SIOG) and European Society of breast cancer specialists (EUSOMA). Lancet Oncol 2012;13:e148-60.

37 Bamber $\mathrm{D}$. The area above the ordinal dominance graph and the area below the receiver operating characteristic graph. J Math Psychol 1975;12:387-415.

38 Vickers AJ, Elkin EB. Decision curve analysis: a novel method for evaluating prediction models. Med Decis Making 2006;26:565-74.

39 Moons KGM, Altman DG, Reitsma JB, et al. Transparent reporting of a multivariable prediction model for individual prognosis or diagnosis (TRIPOD): explanation and ElaborationThe TRIPOD statement: explanation and elaboration. Annals of Internal Medicine 2015;162:W1-73.

40 Rockwood K, Song X, MacKnight C, et al. A global clinical measure of fitness and frailty in elderly people. CMAJ 2005;173:489-95.

41 Cancer Research UK.. Breast cancer incidence (invasive) statistics, 2019. Available: http://www.cancerresearchuk.org/healthprofessional/cancer-statistics/statistics-by-cancer-type/breastcancer/incidence-invasive\#ref-1

42 Reed MWR, Wyld L, Ellis $\mathrm{P}$, et al. Breast cancer in older women: trials and tribulations. Clin Oncol 2009;21:99-102.

43 National Institute for Health and Care Excellence. Nice guidelines (NG101). early and locally advanced breast cancer: diagnosis and treatment. NICE 2018.

44 Morgan J, Richards P, Ward S, et al. Case-Mix analysis and variation in rates of non-surgical treatment of older women with operable breast cancer. Br J Surg 2015;102:1056-63.

45 National Cancer Registration and Analysis Service. COSD v9.0 and pathology Downloads, 2020. Available: http://www.ncin.org. uk/collecting_and_using_data/data_collection/cosd_downloads_ v9

46 Healthcare Quality Improvement Partnership (HQIP). National audit of breast cancer in older patients (NABCOP): annual report 2017.

47 Soong J, Poots AJ, Scott S, et al. Quantifying the prevalence of frailty in English hospitals. BMJ Open 2015:5:e008456.

48 Charlson ME, Pompei P, Ales KL, et al. A new method of classifying prognostic comorbidity in longitudinal studies: development and validation. J Chronic Dis 1987;40:373-83. 